\title{
Shallow donor state of hydrogen in indium nitride
}

\author{
E. A. Davis ${ }^{\text {a) }}$ \\ Department of Physics and Astronomy, University of Leicester, LE1 7RH United Kingdom
}

S. F. J. Cox

ISIS Facility, Rutherford Appleton Laboratory, Chilton, Oxfordshire, OX11 OQX United Kingdom and

Department of Physics and Astronomy, University College London, WCIE 6BT United Kingdom

R. L. Lichti

Department of Physics, Texas Tech University, Lubbock, Texas 79409

C. G. Van de Walle

Palo Alto Research Center, 3333 Coyote Hill Road, Palo Alto, California 94304

(Received 7 October 2002; accepted 27 November 2002)

\begin{abstract}
The nature of the electron states associated with hydrogen in $\mathrm{InN}$ has been inferred by studying the behavior of positive muons, which mimic protons when implanted into semiconductors. The muons capture electrons below $60 \mathrm{~K}$, forming paramagnetic centers with a binding energy of about $12 \mathrm{meV}$. Together with an exceedingly small muon-electron hyperfine constant indicative of a highly delocalized electron wave function, the results confirm the recently predicted shallow-donor properties of hydrogen in InN. C) 2003 American Institute of Physics. [DOI: 10.1063/1.1539547]
\end{abstract}

The role of hydrogen has been crucial to the development of nitride semiconductors for device applications and remains a key issue in material deposition and doping. ${ }^{1}$ Whether present inadvertently or added deliberately, the effect of hydrogen on electronic properties is nearly always significant. Most widely known is its propensity to pair with and passivate other impurities and defects, making isolated defect centers formed by interstitial hydrogen exceedingly difficult to study spectroscopically. Muon implantation is a convenient way of simulating the presence of hydrogen and has proven to be a valuable means of identifying the crystallographic sites and electronic structures of hydrogen defect centers in semiconductors. We refer here specifically to positive muons which, when implanted into nonmetals at low temperatures, can pick up and retain electrons to form muonium, $\mathrm{Mu}=\left[\mu^{+} \mathrm{e}^{-}\right]$, effectively a light isotope of hydrogen. Despite the large isotopic mass ratio, $m_{\mathrm{Mu}} / m_{\mathrm{H}}=1 / 9$, muonium mimics hydrogen rather faithfully in its chemical and electrical interactions with the lattice., ${ }^{2,3}$

The recent discovery ${ }^{4-6}$ that muons implanted into several semiconductors of the II-VI family form shallow donor states was surprising in that investigations conducted over three decades had invariably found muonium to create states lying deep in the semiconducting gap. These deep states are known for $\mathrm{Si}, \mathrm{Ge}$, many III-Vs and indeed some II-VI compounds, e.g., $\mathrm{ZnS}$ and $\mathrm{ZnSe}$. Experiments on muonium in $\mathrm{ZnO}^{5}$ were made in direct response to theoretical work ${ }^{7}$ which predicted that hydrogen should form shallow centers in this material. This result has recently been explicitly confirmed for hydrogen by ENDOR (electron-nuclear double resonance spectroscopy). ${ }^{8}$

The implications for the control of hydrogen as a dopant become particularly topical as these materials are developed for photovoltaic and optoelectronic application. It is, therefore, of importance to understand why hydrogen, and indeed

${ }^{\text {a)} E l e c t r o n i c ~ m a i l: ~ e a d 1 @ ~ @ l e . a c . u k ~}$ other dopants, form extended shallow centers in some semiconductors but compact deep-level centers in others and, if possible, to find criteria for predicting the behavior. We are accumulating evidence that among potentially relevant properties, the depth of the conduction-band minimum below the vacuum level may be a significant parameter. This information is illustrated as a band offset diagram for selected III-V and II-VI materials in Fig. 1. ${ }^{9}$ Rather than use experimental electron affinities, which are sensitive to surface conditions, we use natural band offsets as calculated from first-principles calculations ${ }^{10}$ to line up the band structures.

We now appeal to an observation that, for those materials where it is known, the Fermi level at which $\mathrm{H}^{+}$and $\mathrm{H}^{-}$ defect centers have equal formation probability falls at closely similar depths, again referred to the vacuum level. ${ }^{11}$ For simplicity it is drawn as a constant level for all the materials of Fig. 1. Where this $+/-$ level falls within the forbidden gap, hydrogen and muonium show deep-level amphoteric behavior. In contrast, where the level intersects the

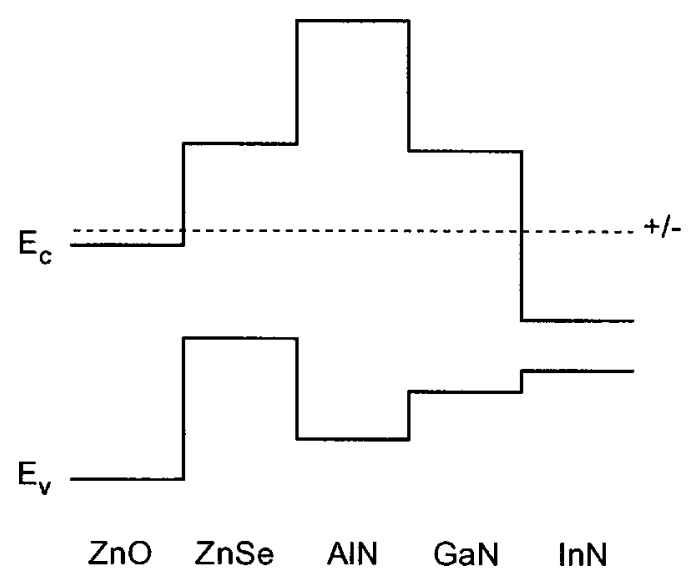

FIG. 1. Approximate position of the hydrogen $+/-$ defect level for the III-V nitrides along with $\mathrm{ZnO}$ and $\mathrm{ZnSe}$, within a band-lineup model. Shallow donor states are predicted for $\mathrm{H}$ and $\mathrm{Mu}$ when this level lies above the conduction-band minimum, as for $\mathrm{ZnO}$ and $\mathrm{InN}$. 


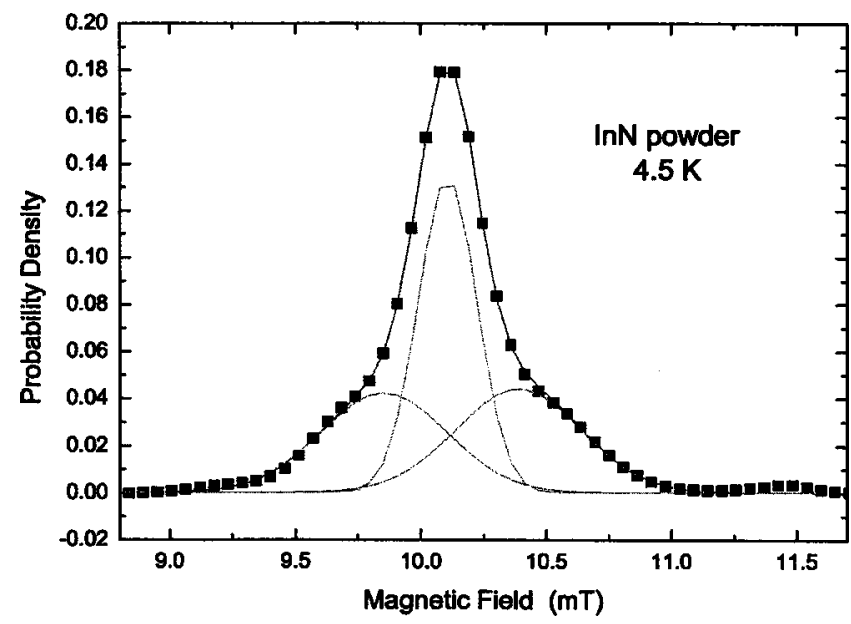

FIG. 2. Muon spin rotation spectrum for $\mathrm{InN}$ at $4.5 \mathrm{~K}$ in an applied field of $10.1 \mathrm{mT}$ obtained from time-domain data by a maximum entropy method. Satellite lines represent the $\mathrm{Mu}^{0}$ state of a shallow donor; the $0.68 \mathrm{mT}$ splitting translates to a hyperfine constant of $92 \mathrm{kHz}$.

conduction band, it is resonant with band states and the electron falls to the conduction-band minimum. This is precisely the condition for weak binding in a classic shallow donor state, i.e., in a greatly dilated hydrogenic orbital determined by electron effective mass and bulk dielectric constant.

Figure 1 implies that, among the II-VIs, for example, the hydrogen $+/-$ level is deep in the gap for $\mathrm{ZnSe}$ but resonant with the conduction band for $\mathrm{ZnO}$. This is supported by explicit density functional calculations for these two materials ${ }^{12}$ and fully borne out by muonium experiments. Within the group-III nitrides, the prediction of Fig. 1 of shallow-donor behavior in InN and contrasting deep-level amphoteric behavior in $\mathrm{GaN}$ and $\mathrm{AlN}$ is likewise explicit in the results of density functional calculations. ${ }^{13}$ In this letter we report experimental confirmation for the existence of a shallow donor state associated with muonium in InN, supporting the prediction of the band-offset model and theory.

Figure 2 shows the frequency spectrum for muon spin precession in InN obtained at the ISIS pulsed muon source using high-purity powder from Alfa Aesar. The spectrum shown here is the frequency transform, plotted in effective field units, of a raw precession signal obtained by a maximum-entropy method. Its unusual line shape and overall width cannot be accounted for solely by dipolar interactions between the muon and adjacent nuclei: the presence of a small but significant Fermi contact interaction, i.e., an electronic contribution, is revealed by deconvolving the spectrum into a dominant central line together with a pair of symmetrically positioned satellites. The central line corresponds to the Larmor precession of those muons that have not captured an electron, whereas the satellite lines correspond to muonium centers, i.e., to muons that have bound an unpaired electron on thermalization. In effect, the hyperfine coupling field either adds to or substracts from the externally applied field, depending on whether the electron is spin up or spin down. The satellite splitting is thus a direct measure of the muonium hyperfine constant; yielding $A=92 \mathrm{kHz}$, a value that is essentially constant for all temperatures where the satellites are discernible.

Figure 3(a) shows the variation of relative amplitudes
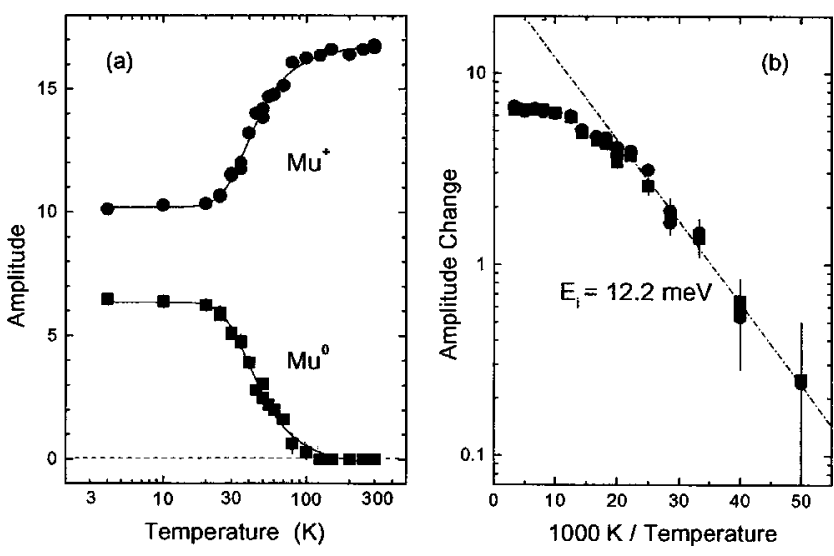

FIG. 3. Results of time-domain fits to the muon spin rotation data: (a) temperature dependent amplitudes of the $\mathrm{Mu}^{0}$ and $\mathrm{Mu}^{+}$signals, and (b) an Arrhenius plot of the amplitude changes across the ionization region.

with temperature through the ionization regime. The curve marked $\mathrm{Mu}^{+}$is related to the amplitude of the central line, representing the diamagnetic or dissociated fraction; the curve marked $\mathrm{Mu}^{0}$ corresponds to the sum of the two satellite amplitudes representing the undissociated or neutral paramagnetic fraction. As the temperature increases, the central line grows at the expense of the satellites, with the total remaining essentially constant. An Arrhenius plot [Fig. 3(b)] of the amplitude changes during ionization yields an asymptotic activation energy of $E_{i} \simeq 12 \mathrm{meV}$.

The experimentally determined hyperfine constant of 92 $\mathrm{kHz}$ is a measure of unpaired electron spin density at the muon. In the hypothesis of an isotropic hydrogenic center, this Fermi contact interaction varies as the inverse cube of the effective Bohr radius, i.e., loosely speaking, as the inverse volume of the electron orbital. Scaling from free atomic muonium in its $1 S$ ground state, for which the hyperfine constant is some four orders of magnitude higher at 4.5 $\mathrm{GHz},{ }^{2}$ we obtain an effective Bohr radius for the extended state in InN of $a^{*}=a_{0} \times(4.5 \mathrm{GHz} / 92 \mathrm{kHz})^{1 / 3}=1.9 \mathrm{~nm}$.

The results are strongly suggestive of a classic effectivemass shallow donor, the reduction in binding energy and dilation of the orbital being due to the dielectric screening of the defect charge and the low effective mass for electron orbitals constructed of conduction-band states. In the usual notation and approximations, and taking $m^{*}=0.12 m_{e}$ and $\epsilon=9.1$ for $\operatorname{InN},{ }^{14}$ the expectations of this model are $R^{*}$ $=R_{0}\left(\mathrm{~m}^{*} / \mathrm{m}_{e}\right) / \epsilon^{2}=20 \mathrm{meV}$ and $a^{*}=a_{0} \epsilon /\left(\mathrm{m}^{*} / \mathrm{m}_{e}\right)=4 \mathrm{~nm}$. The agreement to within a factor of 2 between our results and these estimates, for both parameters, is certainly as good as can be expected in the simplistic effective-mass model.

A pattern is beginning to emerge for the formation of deep and shallow states of interstitial hydrogen in III-V and II-VI compound semiconductors. In the II-VIs, the problem is exemplified by the predicted contrast between deep-level amphoteric behavior in $\mathrm{ZnSe}$ and shallow-donor behavior in $\mathrm{ZnO}{ }^{12}$ In the III-Vs it is illustrated by a similar contrast between GaN or AlN and InN. ${ }^{13}$ The theoretical predictions for hydrogen states are substantially borne out by our experimental studies of their muonium counterparts. ${ }^{15}$

The implication of the results of the present work is that hydrogen is a likely source of $n$-type conductivity in $\mathrm{InN}$. Of interest also is whether, and over what composition range, 
this shallow state persists when $\mathrm{InN}$ is alloyed with other nitrides for device application. Certainly the important consequence of the present experimental results, and their corroboration of theoretical predictions, is that hydrogen must be added to the list of potential dopants in these materials.

This work was supported by the Engineering and Physical Sciences Research Council of the U.K. [GR/R25361 (E.A.D., S.F.J.C.)], the U.S. National Science Foundation [DMR-0102862 (R.L.L.)], the Robert A. Welch Foundation [D-1321 (R.L.L.)], and the Air Force Office of Scientific Research [Award No. F49620-02-1-1163 (C.G.V.d.W.)].

${ }^{1}$ S. J. Pearton and J. W. Lee, in Hydrogen in Semiconductors II, Semicond. and Semimetals Vol. 61, edited by N. Nickel (Academic, San Diego, 1999), pp. 441-478.

${ }^{2}$ B. D. Patterson, Rev. Mod. Phys. 60, 69 (1988).

${ }^{3}$ R. L. Lichti, in Ref. 1, pp. 311-371.

${ }^{4}$ J. M. Gil, H. V. Alberto, R. C. Vilâo, J. Piroto Duarte, P. J. Merdes, L. P. Ferreira, N. Ayres de Campos, A. Weidinger, J. Krauser, Ch. Niedermayer, and S. F. J. Cox, Phys. Rev. Lett. 83, 5294 (1999).
${ }^{5}$ S. F. J. Cox, E. A. Davis, S. P. Cottrell, P. J. C. King, J. S. Lord, J. M. Gil, H. V. Alberto, R. C. Vilâo, J. Piroto Durate, N. Ayres de Capos, A. Weidinger, R. L. Lichti, and S. J. C. Irvine, Phys. Rev. Lett. 86, 2601 (2001)

${ }^{6}$ J. M. Gil, H. V. Alberto, R. C. Vilâo, J. Piroto Duarte, N. Ayres de Campos, A. Weidinger, J. Krauser, E. A. Davis, S. P. Cottrell, and S. F. J. Cox, Phys. Rev. B 64, 075205 (2001).

${ }^{7}$ C. G. Van de Walle, Phys. Rev. Lett. 85, 1012 (2000).

${ }^{8}$ D. M. Hofmann, A. Hofstaetter, F. Leiter, H. Zhou, F. Hereckes, B. K. Meyer, S. B. Orlinskii, J. Schmidt, and P. G. Bararov, Phys. Rev. Lett. 88, 045504 (2002).

${ }^{9}$ Figure 1 reflects the recently revised value of the $\mathrm{InN}$ band gap [J. Wu, W. Walukiewicz, K. M. Yu, J. W. Ager III, E. E. Haller, Hai Lu, W. J. Schaff, Y. Saito, and Y. Nanishi, Appl. Phys. Lett. 80, 3967 (2002)], but our predictions and results were not affected by this revision.

${ }^{10}$ See, e.g., C. G. Van de Walle and J. Neugebauer, Appl. Phys. Lett. 70, 2577 (1997)

${ }^{11}$ C. G. Van de Walle (unpublished).

${ }^{12}$ C. G. Van de Walle, Phys. Status Solidi B 229, 221 (2002).

${ }^{13}$ S. Limpijumnong and C. G. Van de Walle, Phys. Status Solidi B 228, 303 (2001).

${ }^{14}$ Semiconductors-Basic Data, edited by O. Madelung (Springer, New York, 1996).

${ }^{15}$ S. F. J. Cox, R. L. Lichti, and E. A. Davis, J. Phys. D 35, 586 (2002). 